\title{
PHASE SLIP IN AN UNDULATOR WITH POLE AND BPM ERRORS *
}

\author{
P. $\mathrm{Emma}^{\dagger}$ \\ Stanford Linear Accelerator Center, Stanford, CA 94309, USA
}

\begin{abstract}
A statistical analysis of a corrected electron trajectory through a planar FEL undulator is used to predict the optimal beam position monitor (BPM) spacing. The undulator is composed of modular sections, each containing many dipoles with random field and roll angle errors. Located between each section are inaccurate BPMs, steering correctors, and possibly quadrupole magnets. An analytical formula for electron-to-photon phase errors is derived and used to estimate the best BPM spacing. The results are applied to the LCLS FEL undulator [1], which has demanding requirements on electron trajectory straightness.
\end{abstract}

\section{INTRODUCTION}

The requirements on the degree of straightness of an electron trajectory through a SASE-based FEL undulator can be quite demanding. For short wavelength undulator radiation (1.5 ̊ for LCLS) a stringent requirement exists on the relative phase relationship between the electron beam and the radiated photon beam. Undulator field and beam position monitor (BPM) accuracy errors can make the electron beam travel a longer path, creating a phaseshift with respect to the photon beam. Significant phase errors can move the electron beam away from resonance and negate the FEL gain. Since the location and number of BPMs along the undulator is an important factor in achieving a straight trajectory, it is useful to have a simple way to estimate the expected electron-to-photon phase errors and the rms trajectory amplitude, both as a function of BPM separation distance, BPM resolution, and pole field errors. Previous work [2] has examined the effects of pole errors, but without the effects of inaccurate BPMs.

We derive a formula used to estimate the optimal BPM separation along an undulator given BPM resolution and pole field and roll angle errors. Misaligned quadrupole magnets also affect the trajectory, but steering corrections applied at, or near, the quadrupoles can completely compensate this trajectory component. Since the limit of this compensation is solely dependent on the resolution and transverse alignment of the BPMs, we ignore quadrupole misalignments. They are included implicitly in the steering corrections and the treatment of BPM limitations. Each quadrupole is assumed to have a nearby steering corrector and BPM in both planes.

The existence of an optimal spacing can be imagined by considering each type of error (BPM and dipole) in

\footnotetext{
* Work supported by DOE contract DE-AC03-76SF00515.

$\dagger$ Emma@SLAC.Stanford.edu
}

isolation. If the BPMs are inaccurate (misaligned or resolution limited), but the pole fields are perfect, then a closer BPM spacing forces a higher frequency trajectory distortion after steering correction. This produces larger trajectory angles between BPMs and larger phase lag with respect to the radiated photon beam. In contrast, steering correction using perfect BPMs, but imperfect pole fields, will generate a larger cumulative trajectory deviation between BPMs as the BPM spacing is increased, again causing an increased phase lag. BPM errors demand a long BPM spacing, while pole errors suggest a short BPM spacing (for constant undulator period). The goal here is to statistically estimate the optimum spacing, the expected trajectory amplitude, and the mean phase error.

\section{TRAJECTORY ANALYSIS}

A simplified planar undulator section is shown in Figure 1 . The full undulator is many such sections.

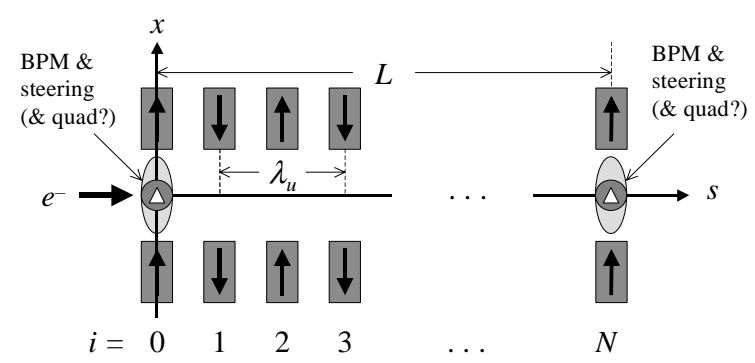

Figure 1. Simplified planar undulator section used to analyze electron trajectory. Section has length $L$ with $N / 2$ periods and a BPM and steering corrector placed between each section. Quadrupole magnets may be located at or near BPMs.

The section length is $L$, the undulator period is $\lambda_{u}$, and there are $N / 2=L / \lambda_{u}$ periods over the section ( $N$ dipoles $)$. A BPM and a steering element (or moveable quadrupole) are placed at each section boundary. For simplicity, this includes no period breaks, and wiggler termination is ignored. Quadrupole magnets, if used, are assumed to be placed at or near the BPMs, but are not a factor since their misalignments simply change the steering corrections required, and the focusing is not relevant for the single particle trajectory. An incoming betatron oscillation is also ignored since it can be removed at the undulator input with steering, or added to these results as an independent effect. It is assumed that no significant focusing exists within the undulator section, but such effects can be added in a modified analysis.

A perfect undulator (and perfect initial trajectory) produce a nominal reference trajectory composed of the small wiggles associated with an undulator. This reference 
is subtracted and only the difference trajectory, produced by pole errors and inaccurate steering, is examined.

For a sinusoidal varying field with peak $B_{0}$ and relative field errors $\left(\Delta B_{j} / B_{0}\right)$, each dipole error adds a transverse kick angle at the center of the $j^{\text {th }}$ dipole of

$$
\theta_{j}=\frac{B_{0} \lambda_{u}}{\pi(B \rho)}\left(\Delta B_{j} / B_{0}\right)=\frac{2 K}{\gamma}\left(\Delta B_{j} / B_{0}\right),
$$

where $(B \rho)$ is the standard magnetic rigidity, $\gamma$ is the Lorentz energy factor, $K$ is the undulator parameter, $K \equiv e B_{0} \lambda_{u} / 2 \pi m c, m$ is the electron rest mass, $c$ is the speed of light, and $e$ is the electron charge. Ignoring any weak focusing of the undulator, each upstream kick at location $j(0 \leq j<N)$ displaces the electron beam at a downstream location $i \quad(j<i \leq N)$ by $\Delta x_{i j}=\theta_{j}\left(s_{i}-s_{j}\right)$ $=\lambda_{u} \theta_{j}(i-j) / 2$. The sum over all upstream displacements, $\Delta x_{i j}$; plus the displacement, $\theta_{c} \lambda_{u} i / 2$, produced by an initial beam angle, $\theta_{c}$, at $j=0$; plus an initial BPM-limited position, $b_{1}$, produces a trajectory position at location $i$ of

$$
x_{i}=\frac{\lambda_{u}}{2}\left[\theta_{c} i+\sum_{j=0}^{i} \theta_{j}(i-j)\right]+b_{1} .
$$

The angle $\theta_{c}$ is the sum of $(a)$ an incoming angle from the previous section, plus $(b)$ a correction angle used to steer the trajectory at the next BPM (at section's end). The offset $b_{1}$ is the initial $e^{-}$beam position at $j=0$ resulting from upstream steering of the trajectory to the first BPM offset, $b_{1}$. The BPM offset can be interpreted as a transverse misalignment, a noise component, or both.

The angle, $\theta_{c}$, is defined by steering such that the next BPM 'reads' zero. Since this next BPM has a different random offset, $b_{2}$, than the first BPM, the steering correction will produce $x_{N}=b_{2}$. Eq. (2) is used with $i=N$ and $x_{N}=b_{2}$, to solve for $\theta_{c}$. This is then substituted back into (2) and after rearrangement is put into a form where one sum extends from $j=0$ to $i-1$, and a second sum extends from $j=i$ to $\mathrm{N}$. The steered trajectory at any section location, $i$, is then given by

$$
x_{i}=\frac{\lambda_{u}}{2 N}\left[(i-N) \sum_{j=0}^{i-1} j \theta_{j}-i \sum_{j=i}^{N} \theta_{j}(N-j)\right]+\left(1-\frac{i}{N}\right) b_{1}+\frac{i}{N} b_{2} .
$$

This is used to calculate the trajectory and phase slip over an ensemble of undulator sections with random errors.

\section{PHASE SLIP ERRORS}

The path length difference of an electron with speed $v=c$ and angle $x^{\prime}(\ll<1)$, with respect to a photon with zero angle, over a length $l$, is given by $\Delta s=l\left(1+x^{\prime 2}\right)^{1 / 2}-l \approx l x^{\prime 2} / 2$. The beam angle, $x_{i}^{\prime}$, through the $i^{\text {th }}$ pole to pole separation of length, $\lambda_{u} / 2$, is $x_{i}^{\prime}=2\left(x_{i+1}-x_{i}\right) / \lambda_{u}$, where we approximate small kicks over a dipole as equivalent thin-kicks at pole centers. This approximation is verified in numerical studies.

The positions $x_{i}$ and $x_{i+1}$ are taken from Eq. (3) and after some rearrangement, the beam angle $x_{i}^{\prime}$ is written as

$$
x_{i}^{\prime}=\frac{1}{N}\left[\sum_{j=1}^{N} j \theta_{j}-N \sum_{j=i+1}^{N} \theta_{j}+\frac{2}{\lambda_{u}}\left(b_{2}-b_{1}\right)\right] .
$$

The electron-to-photon 'phase' error at location $i$ is $\Delta \varphi_{i}=k_{r} \Delta s_{i}=k_{r} \lambda_{u} x^{\prime 2} / 4$, where $\lambda_{r}$ is the FEL radiation wavelength and $k_{r}=2 \pi / \lambda_{r}$ is the radiation wavenumber. The total phase error over the section (after steering using inaccurate BPMs) is the sum of individual phase errors.

$$
\varphi_{s}=\frac{k_{r} \lambda_{u}}{4 N^{2}} \sum_{i=1}^{N}\left\{\sum_{j=1}^{N} j \theta_{j}-N \sum_{j=i+1}^{N} \theta_{j}+\frac{2}{\lambda_{u}}\left(b_{2}-b_{1}\right)\right\}^{2} \text {. }
$$

We now move toward a statistical analysis of the trajectory and therefore consider $b_{1}, b_{2}$, and the set of angles $\theta_{j}$ as random, uncorrelated, zero-mean variables, where $\left\langle b_{1}{ }^{2}\right\rangle=\left\langle b_{2}{ }^{2}\right\rangle \equiv\left\langle b^{2}\right\rangle$, etc. This is an important step. It implies that the BPM errors used here are the uncorrelated, random component of the relative misalignments of two BPMs separated by one undulator section. This includes static, relative, uncorrelated misalignments as well as BPM readback noise. It does not include, nor should it include, any relative misalignment on a scale longer than an undulator section or any correlated component of misalignment. Realistic simulations of beam-based alignment (BBA) [3], over an undulator indicate that the relative, uncorrelated, random misalignment of two adjacent BPMs can be reduced to very nearly the fundamental BPM noise level. We, therefore, do not need to evaluate BPM alignment with respect to a straight line over the length of the entire undulator. We assume that BBA has been performed adequately, which is a typical requirement for SASE saturation with very short wavelength FELs.

The mean phase error, $\left\langle\varphi_{s}\right\rangle$ (i.e., averaged over many random undulator sections) in Eq. (5) is now reduced using standard relations for the sums of powers of integers. The mean total phase error per section is

$$
\left\langle\varphi_{s}\right\rangle=k_{r}\left[\frac{\lambda_{u}}{24}\left(N^{2}+5\right)\left\langle\theta^{2}\right\rangle+\frac{2}{N \lambda_{u}}\left\langle b^{2}\right\rangle\right] .
$$

The error over the entire undulator length, $L_{u}\left(=N_{s} L\right)$, composed of $N_{s}$ sections, is $\langle\varphi\rangle=\left\langle\varphi_{s}\right\rangle L_{u} / L$, or

$$
\langle\varphi\rangle \approx k_{r} L_{u}\left[\frac{L}{6 \lambda_{u}}\left\langle\theta^{2}\right\rangle+\frac{1}{L^{2}}\left\langle b^{2}\right\rangle\right],
$$

where we assume $N^{2}>$ 5. So far, this result includes trajectory errors in the wiggle-plane only.

Equation (7) shows the tradeoffs anticipated in the choice of an optimum section length, $L$. The section length appears in the numerator of the pole error term (i.e., the $\left\langle\theta^{2}\right\rangle$ term), but in the denominator of the BPM resolution term (i.e., the $\left\langle b^{2}\right\rangle$ term). Therefore, as described in the introduction, a shorter section length increases the phase lag induced by BPM errors, but decreases the phase lag induced by pole errors (for 
constant period). The behavior of Eq. (7), per meter of undulator, is shown in Figure 2. Data points shown with error bars are a Monte Carlo computer calculation [4] at $\left(\Delta B / B_{0}\right)_{\text {rms }}=0.10 \%$ over 1000 undulator sections, used for numerical confirmation of Eq. (7).

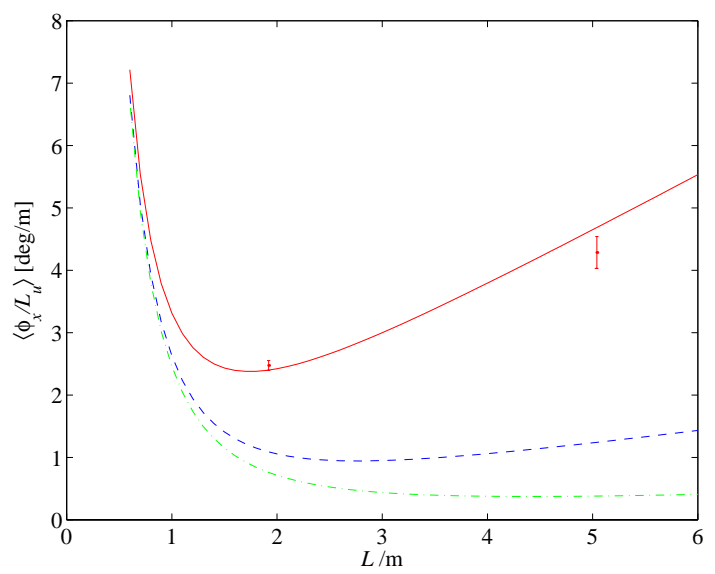

Figure 2. Mean phase error per meter of undulator [Eq. (7)] vs. section length, $L$, for BPM resolution $\left\langle b^{2}\right\rangle^{1 / 2}=1 \mu \mathrm{m}$, and pole errors $\left(\Delta B / B_{0}\right)_{\mathrm{rms}}=0.1 \% \quad$ (solid/red), $0.05 \% \quad$ (dash/blue), and $0.025 \%$ (dash-dot/green), with $\lambda_{u}=3 \mathrm{~cm}, \quad \lambda_{r}=1.5 \AA, \quad K=3.7$, $m c^{2}=14.3 \mathrm{GeV}$. Points w/error bars are tracking simulations.

The result of Eq. (7) can be differentiated with respect to $L$ and set equal to zero in order to calculate the optimal BPM spacing, $L_{\text {opt }}$. In addition, Eq. (1) is used to make the result explicit in terms of the relative pole field errors.

$$
L_{o p t} \approx\left(\frac{3 \gamma^{2} \lambda_{u}\left\langle b^{2}\right\rangle}{K^{2}\left\langle\left(\Delta B / B_{0}\right)^{2}\right\rangle}\right)^{1 / 3}
$$

For an expected level of random, uncorrelated pole errors, and BPM resolution, the optimum BPM spacing can be predicted using Eq. (8).

This can be extended into both the $x$ and $y$ planes by adding up the phase lag values per plane, $\left\langle\varphi_{x y}\right\rangle=\left\langle\varphi_{x}\right\rangle+\left\langle\varphi_{y}\right\rangle$. The additional phase lag due to vertical trajectory errors is ascribed to small random pole rollangle errors, $\psi$. The total mean phase lag over the undulator, including both pole field and pole roll errors, is

$$
\left\langle\varphi_{x y}\right\rangle \approx 2 k_{r} L_{u}\left\{\frac{K^{2} L}{3 \gamma^{2} \lambda_{u}}\left[\left\langle\left(\Delta B / B_{0}\right)^{2}\right\rangle+\left\langle\psi^{2}\right\rangle\right]+\frac{1}{L^{2}}\left\langle b^{2}\right\rangle\right\} .
$$

Eq. (9) is simply twice that of Eq. (7) when $\left\langle\left(\Delta B / B_{0}\right)^{2}\right\rangle=\left\langle\psi^{2}\right\rangle$. In this case, the optimal spacing of Eq. (8) is still valid. From this point on we treat the case where $\left\langle\psi^{2}\right\rangle=\left\langle\left(\Delta B / B_{0}\right)^{2}\right\rangle$, for simplicity. Now substituting $L_{\text {opt }}$ from Eq. (8) into $L$ of Eq. (9) gives the minimum mean phase lag achieved at $L=L_{\text {opt }}$.

$$
\left\langle\varphi_{x y}\right\rangle_{\min }=2 k_{r} L_{u}\left(\frac{3 K^{4}}{\gamma^{4}} \frac{\left\langle b^{2}\right\rangle}{\lambda_{u}^{2}}\left\langle\left(\Delta B / B_{0}\right)^{2}\right\rangle^{2}\right)^{1 / 3}
$$

If Eq. (10) is set to $\leq 2 \pi$ as a reasonable upper limit, the tolerable field errors (and roll errors) are calculated, given the BPM resolution and radiation wavelength, $\lambda_{r}$, using

$$
\left\langle\left(\Delta B / B_{0}\right)^{2}\right\rangle_{\text {tol }}^{1 / 2} \leq \frac{\gamma}{K} \sqrt{\frac{3 \lambda_{u}}{8\left\langle b^{2}\right\rangle^{1 / 2}}\left(\frac{2 \lambda_{r}}{3 L_{u}}\right)^{3 / 4} .}
$$

This result is re-substituted back into Eq. (8) and the optimal BPM spacing is calculated from knowledge only of the BPM resolution or only the field (and roll) errors.

$$
L_{o p t}=\sqrt{\frac{6 L_{u}}{\lambda_{r}}}\left\langle b^{2}\right\rangle^{1 / 2}, L_{o p t}=\frac{\lambda_{r} \lambda_{u} \gamma^{2}}{2 K^{2} L_{u}\left\langle\left(\Delta B / B_{0}\right)^{2}\right\rangle} .
$$

In this case, the pole field (and roll) tolerances are related to the BPM resolution by Eq. (11). The undulator design can therefore be initiated using just the knowledge of the expected BPM resolution. Additional trajectory analysis and more details are available [5].

\section{TRAJECTORY AMPLITUDE}

The rms trajectory amplitude can be similarly analyzed producing Eq. (13). Numerical simulations have also been used to verify the amplitude [5].

$$
\left\langle x_{r m s}^{2}\right\rangle_{s}^{1 / 2} \approx \frac{2}{3} \sqrt{\frac{L^{3} K^{2}}{5 \lambda_{u} \gamma^{2}}\left\langle\left(\Delta B / B_{0}\right)^{2}\right\rangle+\frac{3}{2}\left\langle b^{2}\right\rangle} .
$$

\section{CONCLUSIONS}

For an FEL undulator composed of separate sections bounded by BPMs, steering correctors, and quadrupoles, the undulator section length can be optimized analytically using Eq. (12), from the expected BPM resolution or pole field and roll errors. This assumes beam-based alignment is used to align BPMs with respect to their nearest neighbors, to the level of their resolution. Well controlled pole errors, as produced using shimming methods, will increase the optimal section length by reducing the pole field and roll errors. The mean beta function and its variation, however, must also be considered.

\section{ACKNOWLEDGEMENTS}

We thank Ilan Ben-Zvi and Max Cornacchia for encouragement, and Heinz-Dieter Nuhn for insight.

\section{REFERENCES}

[1] LCLS Design Study Report, SLAC-R-521, April 1998.

[2] L.-H. Yu, et. al., Effect of Wiggler Errors on FEL Gain, Phys. Rev. A, Vol. 45, No. 2, Jan. 15, 1992.

[3] P. Emma, et. al., Beam Based Alignment For The LCLS FEL Undulator, Proceedings of the 1998 Free Electron Laser Conference, Newport News, Virginia, Aug. 1998.

[4] These particle tracking calculations were performed using the computer code Elegant written by M. Borland at ANL.

[5] P. Emma, LCLS-TN-0014, Oct 2000. 